$\mathbb{T}$ periodica polytechnica

\author{
Transportation Engineering \\ $36 / 1-2(2008)$ 87 92 \\ doi: 10.3311/pp.tr.2008-1-2.16 \\ web: http://www.pp.bme.hu/tr \\ (c) Periodica Polytechnica 2008
}

RESEARCH ARTICLE

\section{Robustness properties of the}

hierarchical passivity based formation

control

Tamás Péni

Received 2007-03-03

\begin{abstract}
In this paper the robustness analysis of the hierarchical formation stabilization control proposed by [7] is performed. The analysis is based on the nonlinear small gain theorem and exploits the strict passivity of the components in the closed loop dynamics. The computations are tested via a formation control problem of road vehicles.
\end{abstract}

\author{
Keywords \\ formation control · dynamic inversion · passivity · robust con- \\ trol
}

\section{Acknowledgement}

This work has been supported by the Hungarian Science Fund (OTKA) through grant K60767 and the Hungarian National Office for Research and Technology through the project "Advanced Vehicles and Vehicle Control Knowledge Center" (No: OMFB01418/2004) which is gratefully acknowledged.

\section{Tamás Péni}

Systems and Control Laboratory, Computer and Automation Research Instititute of Hungarian Academy of Sciences, Kende u. 13-17, H-1111 Budapest, Hungary e-mail: pt@scl.sztaki.hu

\section{Introduction}

In the last years the increased computational capabilities of computer systems and the rapid development of the communication and sensor technologies have increased the interest in highly automated unmanned vehicles, which are able to cooperate with other vehicles and are able to perform, in the presence of uncertainties, disturbances and faults, complex tasks beyond the ability of the individual vehicles. This general concept has been realized in multiple applications [2]: Unmanned Aerial Vehicles (UAV-s), [1], Autonomous Underwater Vehicles (AUV-s) and automated highway systems (AHS) [12].

Although the application fields listed above are very different, in the control design several common points can be found. The control of autonomous vehicle groups is generally hierarchical, where the components on the lower levels are local, in the sense that they depend on the particular - and generally nonlinear - vehicle dynamics. These local controllers modify the original vehicle dynamics so that the dynamic behaviour of the closed loop can be modelled by a more simpler - e.g. linear - system. This simple model, which can even be the same for different vehicles, is then used in the design of the higher-level control components, where the prescribed cooperative tasks are taken into consideration. Due to this decoupling the complex, task-dependent control problems have to be solved for simplified vehicle models only, and the controllers obtained will be independent from the real vehicle dynamics. For the design of the high-level cooperative control several methods exist, depending on the prescribed task, the number of vehicles and the design constraints to be satisfied. In this paper we are focusing on the methods based on artificial potential functions [4, 5, 10]. These methods construct a special potential energy function, which takes its minimum at the solution of the cooperative problem. Starting from an arbitrary initial state the controller then tries to steer the system along the gradient of the potential function until the energy reaches its minimum.

It is clear that the stability of the entire hierarchically controlled formation is a key issue in the controller design. Despite of this, the cooperative control literature concentrates mainly on the high-level control design and does not analyse the stability 
properties of the coupled system. Therefore in [7] we have proposed a hierarchical formation stabilization method (consisting of a dynamic inversion based low-level and a passivity based high-level controller) which is able to guarantee the stability of the entire formation. In this paper we concentrate on the robustness properties of this control structure.

The paper is organized as follows. In section 2 the outline of the hierarchical control structure discussed in [7] is presented. In section 3 the robustness properties of the control method is analysed. Section 4 gives a demonstrative example for the calculations and in 5 the most important conclusions are drawn.

\section{Hierarchical passivity based control}

In order to discuss the robustness properties of the method proposed in [7], we have to introduce it briefly. The aim of the control is the stabilization of the formation of vehicles having nonlinear dynamics. The control structure consists of two levels: the dynamic inversion based low-level controller linearizes - at least partially - the nonlinear vehicle dynamics. After the linearization the vehicle can be considered as a simple double integrator, for which the high-level formation controller can be easily designed. In order to have a Lyapunov function proving the stability of the entire closed-loop system a passivity based external feedback is constructed.

\subsection{Vehicle modell}

We assume that the vehicle can be modelled by the following nonlinear dynamic equations:

$$
\begin{aligned}
& \dot{x}_{1}=h\left(x_{1}, x_{2}, t\right) \\
& \dot{x}_{2}=A_{2}(\rho) x_{3}+B_{2}(\rho) u+f(t) \\
& \dot{x}_{3}=A_{3}(\rho) x_{3}+B_{3}(\rho) u
\end{aligned}
$$

where $x_{1}$ is the position of the vehicle and $x_{2}, x_{3}$ are further state variables, depending on the vehicle model used. It has already been shown in [7], that the single track vehicle model expressed in a moving coordinate frame can be rewritten in the form above.

\subsection{Dynamic inversion based low-level controller design}

The low-level part of the hierarchical control framework is based on the dynamic inverse of the vehicle model. The dynamic inverse can be obtained by applying the state transformation $z_{1}=x_{1}=y, z_{2}=\dot{x}_{1}=h\left(x_{1}, x_{2}\right), z_{3}=x_{3}$ to (1) and expressing the control input from the dynamic equation of $z_{2}$. (For the details see [8]). Applying the same argument as [8] the dynamic inversion based controller can be obtained in the following form:

$$
\begin{aligned}
u_{c}= & B_{2}^{-1} J_{x_{2}}^{-1}\left(-J_{x_{1}} z_{2}-J_{x_{2}} A_{2} z_{3 c}-J_{x_{2}} f(t)-J_{t}+v\right) \\
\dot{z}_{3 c}= & A_{3} z_{3 c}+B_{3} u-w= \\
= & A_{3} z_{3 c}-B_{3} B_{2}^{-1} J_{x_{2}}^{-1} J_{x_{1}} z_{2}-B_{3} B_{2}^{-1} A_{2} z_{3 c}- \\
& B_{3} B_{2}^{-1} f(t)-B_{3} B_{2}^{-1} J_{x_{2}}^{-1} J_{t}+ \\
& B_{3} B_{2}^{-1} J_{x_{2}} v-w=\left(A_{3}-B_{3} B_{2}^{-1} A_{2}\right) z_{3 c}+u_{c}^{*}-(\text { (2) }
\end{aligned}
$$

where $J_{x_{2}}=\frac{\partial h}{\partial x_{2}}=\left[\begin{array}{cc}-v \sin \theta & \cos \theta \\ v \cos \theta & \sin \theta\end{array}\right], J_{x_{1}}=\frac{\partial h}{\partial x_{1}}, J_{t}=\frac{d h}{d t}$ and $v$ and $w$ are additional control inputs defined later and $z_{3 c}$ is the inner state of the controller used to estimate the unmeasured state $z_{3}$. The controller above transforms the original vehicle dynamics into the following partially linear closed-loop system:

$$
\begin{array}{rc}
\dot{z}_{1} & =z_{2} \\
\dot{z}_{2} & =v+J_{x_{2}} A_{2}\left(z_{3}-z_{3 c}\right) \\
\dot{z}_{3}-\dot{z}_{3 c} & =A_{3}\left(z_{3}-z_{3 c}\right)+w
\end{array}
$$

which, apart from the dynamics of the approximation error $z_{3}-z_{3 c}$, is equivalent to a double-integrator. The nonlinearity is caused by the parameter-dependence of matrices $A_{2}, A_{3}$ and state dependence of $J_{x_{2}}$. We have shown in [7] that the controller above is applicable if the error dynamics $\dot{e}_{3}=A_{3} e_{3}$ is quadratically stable with Lyapunov function $W\left(e_{3}, \rho\right)=e_{3}^{T} W(\rho) e_{3}$.

\subsection{High-level formation control design}

The goal of the high-level controller is to solve the formation control problem, i.e. to steer the group of vehicles into a prescribed spatial formation, while the entire group follows a predefined trajectory. This problem class comprises several special cooperative control problems, e.g. geometric formation shaping, obstacle avoidance or coordinated collective motion of high number of vehicles called 'flocking' [4]. Since the low-level controller has already linearized the dynamics, the high-level controller can be implemented as if the vehicles had double integrator dynamics.

Assume that the formation control problem is prescribed for a group of $N$ vehicles. Suppose that this problem can be solved by using artificial potential function, i.e. there exists an artificial potential function $V\left(\zeta_{1}\right), \zeta_{1}=\left[z_{1}^{1}, z_{1}^{2}, \ldots, z_{1}^{N}\right]$ so that $V\left(\zeta_{1}\right)$ has global minimum at the prescribed spatial formation. Consider now, the total energy of the point-mass system:

$$
\mathcal{V}\left(\zeta_{1}, \zeta_{2}\right)=V\left(\zeta_{1}\right)+\frac{1}{2}\left\|\zeta_{2}\right\|^{2}
$$

where $\zeta_{2}=\left[z_{2}^{1}, z_{2}^{2}, \ldots, z_{2}^{N}\right]$. Let the control input $v_{c}$ be chosen as follows:

$$
\begin{aligned}
v_{c}\left(\zeta_{1}, \zeta_{2}\right) & =-\frac{\partial V\left(\zeta_{1}\right)}{\partial \zeta_{1}}-k \zeta_{2} \quad k>0 \\
v_{c}^{i} & =-\frac{\partial V\left(z_{1}^{i}\right)}{\partial z_{1}^{i}}-k z_{2}^{i}
\end{aligned}
$$

It can be easily checked that this feedback stabilizes the formation by rendering the time derivative of $\mathcal{V}\left(\zeta_{1}, \zeta_{2}\right)$ negative:

$$
\dot{\mathcal{V}}\left(\zeta_{1}, \zeta_{2}\right)=\frac{\partial V}{\partial \zeta_{1}} \zeta_{2}-\zeta_{2}^{T} \frac{\partial V}{\partial \zeta_{1}}-k \zeta_{2}^{T} \zeta_{2}=-k\left\|\zeta_{2}\right\|^{2} \leq 0
$$

In order to calculate (??) every vehicle has to know the position and velocity of the others. This information has to be shared via appropriate communication channels. 


\subsection{Passivity based external feedback design}

Now, being in possession of the high-level and the low-level controllers we can build up the hierarchical control structure. For this, let us substitute $v_{c}\left(\zeta_{1}, \zeta_{2}\right)$ into (3) to get the coupled vehicle dynamics:

$$
\begin{aligned}
& \dot{\zeta}_{1}=\zeta_{2} \\
& \dot{\zeta}_{2}=v_{c}\left(\zeta_{1}, \zeta_{2}\right)+\mathcal{A}_{2} \varepsilon_{3} \\
& \dot{\varepsilon}_{3}=\mathcal{A}_{3} \varepsilon_{3}+\omega
\end{aligned}
$$

where

$$
\begin{aligned}
& \mathcal{A}_{2}=\operatorname{diag}\left(J_{x_{2}^{1}}^{1} A_{2}^{1}, \ldots, J_{x_{2}^{N}}^{N} A_{2}^{N}\right), \\
& \mathcal{A}_{3}=\operatorname{diag}\left(\bar{A}_{3}^{1}, \ldots, \bar{A}_{3}^{N}\right), \varepsilon_{3}=\left[e_{3}^{1}, \ldots, e_{3}^{N}\right] \\
& \text { and } \omega=\left[w^{1}, \ldots, w^{N}\right] .
\end{aligned}
$$

Notice that the equations (7) realizes a partial interconnection of the following two subsystems:

$$
\begin{aligned}
& \text { 1. } \dot{\varepsilon_{3}}=\mathcal{A}_{3} \varepsilon_{3}+w \\
& \text { 2. } \dot{\zeta}_{1}=\zeta_{2} \\
& \zeta_{2}=v_{c}\left(\zeta_{1}, \zeta_{2}\right)
\end{aligned}
$$

Our aim is to choose the external control input $w$ in such a way that a Lyapunov function can be constructed for the entire controlled system. We solve this problem by using passivity-based technique in the following way: first new inputs and outputs are chosen for the subsystems with respect to which they will be passive. Then the control input $w$ is set so that the dynamics (7) realize a negative feedback interconnection of the subsystems, which consequently will be asymptotically stable [11].

Since Subsystem 2 is asymptotically stable with Lyapunov function $\mathcal{V}\left(\zeta_{1}, \zeta_{2}\right)$, then by calculating the time derivative of $\mathcal{V}$ we get hints for the choice of input $u_{2}$ and output $y_{2}$ :

$$
\begin{aligned}
\frac{d \mathcal{V}}{d t} & =\underbrace{\frac{\partial \mathcal{V}\left(\zeta_{1}, \zeta_{2}\right)}{\partial \zeta_{1}} \zeta_{2}+\frac{\partial \mathcal{V}\left(\zeta_{1}, \zeta_{2}\right)}{\partial \zeta_{2}} v_{c}}_{<0}+\underbrace{\frac{\partial \mathcal{V}\left(\zeta_{1}, \zeta_{2}\right)}{\partial \zeta_{2}} \mathcal{A}_{2}}_{y_{2}^{T}} \underbrace{\varepsilon_{3}}_{u_{2}}= \\
& -k\left\|\zeta_{2}\right\|^{2}+y_{2}^{T} u_{2} \leq y_{2}^{T} u_{2}
\end{aligned}
$$

i.e. the subsystem 2 is passive with storage function $\mathcal{V}$. A similar input/output selection procedure can be carried out for the subsystem 1 by introducing the Lyapunov function $\mathcal{W}\left(\varepsilon_{3}\right)=$ $\frac{1}{2} \varepsilon_{3}^{T} \mathcal{W} \varepsilon_{3}, \mathcal{W}=\operatorname{diag}\left(W^{1}, \ldots, W^{N}\right)$ :

$$
\begin{aligned}
\frac{d \mathcal{W}\left(\varepsilon_{3}\right)}{d t}=\quad & \underbrace{\varepsilon_{3}^{T} \mathcal{W} \mathcal{A}_{3}}_{y_{1}^{T}} \varepsilon_{3}<0 \\
& -\lambda^{*}\left\|\varepsilon_{3}\right\|^{2}+y_{1}^{T} u_{1} \leq y_{1}^{T} u_{1}
\end{aligned}
$$

where

$$
\lambda^{*}=\frac{1}{2} \min _{\rho} \underline{\lambda}\left(-\mathcal{W} \mathcal{A}_{3}(\rho)-\mathcal{A}_{3}(\rho)^{T} \mathcal{W}\right)>0
$$

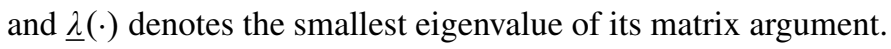
So, the subsystem 1 is also passive with respect to the chosen input $u_{1}$ and output $y_{1}$ with storage function $\left(\mathcal{W}\left(e_{3}\right)\right)$.

Notice that the partial interconnection of subsystem 1 and 2, coming from the original structure (7), can be expressed by the following relation $u_{2}=y_{1}$. (The interconnected structure is depicted in Fig. 11 In order to achieve the negative feedback interconnection we have to set $u_{1}=-y_{2}$ as it can be seen in Fig. 1. This means that the external control input $\omega$ has to be chosen as follows:

$$
\begin{array}{cc}
\omega= & -\mathcal{W}^{-1} \mathcal{A}_{2}^{T} \frac{\partial \mathcal{V}\left(\zeta_{1}, \zeta_{2}\right)}{\partial \zeta_{2}}=-\mathcal{W}^{-1} \mathcal{A}_{2}^{T} \zeta_{2} \\
\text { or } & \\
w^{i}= & -\left(W^{i}\right)^{-1} A_{2}^{T}\left(J_{x_{2}}^{i}\right)^{T} z_{2}^{i}
\end{array}
$$

To prove the asymptotic stability of the entire system we prove first that the interconnected system is passive with storage function $S\left(\zeta_{1}, \zeta_{2}, \varepsilon_{3}\right)=\mathcal{V}\left(\zeta_{1}, \zeta_{2}\right)+\mathcal{W}\left(\varepsilon_{3}\right)$ and then we will see that this function can serve as Lyapunov function in our special case. Let us introduce two new, external inputs denoted by $u_{e 1}$ and $u_{e 2}$ respectively according to Fig. 1. By calculating the time-derivative of $S\left(\zeta_{1}, \zeta_{2}, \varepsilon_{3}\right)$

$$
\begin{gathered}
\dot{S}=\frac{d}{d t}\left\{\mathcal{V}\left(\zeta_{1}, \zeta_{2}\right)+\mathcal{W}\left(\varepsilon_{3}\right)\right\}=\underbrace{\frac{\partial \mathcal{V}}{\partial \zeta_{1}} \zeta_{2}+\frac{\partial \mathcal{V}}{\partial \zeta_{2}} v_{c}}_{<0}+ \\
\underbrace{\varepsilon_{3}^{T} \mathcal{W} \mathcal{A}_{3} \varepsilon_{3}}_{<0}+y_{2}^{T} u_{2 e}+y_{1}^{T} u_{1 e} \leq\left[\begin{array}{ll}
y_{1}^{T} & y_{2}^{T}
\end{array}\right]\left[\begin{array}{l}
u_{1 e} \\
u_{2 e}
\end{array}\right]
\end{gathered}
$$

we can see that the interconnected system is passive with respect to input $\left[\begin{array}{l}u_{1 e} \\ u_{2 e}\end{array}\right]$ and output $\left[\begin{array}{l}y_{1} \\ y_{2}\end{array}\right]$ with storage function $S\left(\zeta_{1}, \zeta_{2}, \varepsilon_{3}\right)$. In our case the external inputs $u_{e 1}$ and $u_{e 2}$ are 0 thus $\dot{\mathcal{V}}\left(\zeta_{1}, \zeta_{2}\right)+\dot{\mathcal{W}}\left(\varepsilon_{3}\right) \leq 0$. The immediate consequence of this result is that the positive definite function $\mathcal{V}\left(\zeta_{1}, \zeta_{2}\right)+\mathcal{W}\left(\varepsilon_{3}\right)$ is an appropriate Lyapunov function for the coupled dynamics (7). (For the details see [7].)

\section{Robustness properties of the controlled system}

In the possession of the Lyapunov function $\mathcal{V}\left(\zeta_{1}, \zeta_{2}\right)+\mathcal{W}\left(\varepsilon_{3}\right)$ we can determine a class of perturbation models, against which, the stability of the hierarchically controlled system is preserved. For this, let the disturbances $\delta_{1}, \delta_{2}$ be added to the dynamic equations (1) as follows:

$$
\begin{aligned}
& \dot{x}_{1}=h\left(x_{1}, x_{2}, t\right) \\
& \dot{x}_{2}=A_{2}(\rho) x_{3}+B_{2}(\rho) u+f(t)+\delta_{1} \\
& \dot{x}_{3}=A_{3}(\rho) x_{3}+B_{3}(\rho) u+\delta_{2}
\end{aligned}
$$

By applying the state transformation and control input (5) to the group of $N$ vehicles above, the coupled dynamics (7) of the controlled system can be given by

$$
\begin{aligned}
& \dot{\zeta}_{1}=\zeta_{2} \\
& \dot{\zeta}_{2}=v_{c}\left(\zeta_{1}, \zeta_{2}\right)+\mathcal{A}_{2} \varepsilon_{3}+J d_{1} \\
& \dot{\varepsilon}_{3}=\mathcal{A}_{3} \varepsilon_{3}+\omega+d_{2}
\end{aligned}
$$

where $d_{1}=\left[\delta_{1}^{1}, \ldots, \delta_{1}^{N}\right], d_{2}=\left[\delta_{2}^{1}, \ldots, \delta_{2}^{N}\right]$ and $J=$ $\operatorname{diag}\left(J_{x_{2}^{1}}^{1}, \ldots, J_{x_{2}^{N}}^{N}\right)$. Calculate now the time derivative of the 



Fig. 1. Interconnection of passive subsystems (left). The hierarchically controlled system completed with perturbation $\Delta$ (right)

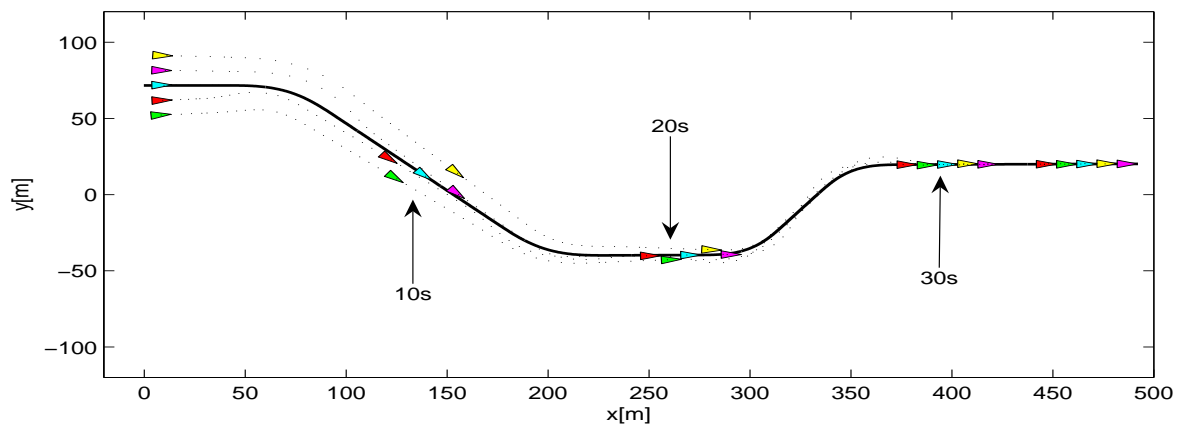

Fig. 3. Simulation results. The motion of the vehicles along the prescribed trajectory.

Lyapunov function $\mathcal{V}\left(\zeta_{1}, \zeta_{2}\right)+\mathcal{W}\left(\varepsilon_{3}\right)$ :

$$
\begin{gathered}
\frac{d}{d t}\left\{\mathcal{V}\left(\zeta_{1}, \zeta_{2}\right)+\mathcal{W}\left(\varepsilon_{3}\right)\right\}= \\
-k\left\|\zeta_{2}\right\|^{2}-\lambda^{*}\left\|\varepsilon_{3}\right\|^{2}+\zeta_{2}^{T} J d_{1}+\varepsilon_{3}^{T} W d_{2} \\
\leq-\alpha \underbrace{\left[\begin{array}{ll}
\zeta_{2}^{T} & \varepsilon_{3}^{T}
\end{array}\right]}_{y^{T}} \underbrace{\left[\begin{array}{c}
\zeta_{2} \\
\varepsilon_{3}
\end{array}\right]}_{y}+\underbrace{\left[\begin{array}{ll}
\zeta_{2}^{T} & \varepsilon_{3}^{T}
\end{array}\right]}_{y^{T}} \underbrace{\left[\begin{array}{l}
J d 1 \\
W d_{2}
\end{array}\right]}_{u}
\end{gathered}
$$

where $\alpha=\min \left(k, \lambda^{*}\right)$. It can be seen that the controlled system is strictly output passive with respect to output $y=\left[\begin{array}{ll}\zeta_{2}^{T} & \varepsilon_{3}^{T}\end{array}\right]$ and input $u=\left[\begin{array}{ll}\left(J d_{1}\right)^{T} & \left(W d_{2}\right)^{T}\end{array}\right]^{T}$. We know from [11] that all strictly output passive systems have finite $L_{2}$ gain. In our case the $L_{2}$ gain is $\frac{1}{\alpha}$, i.e.:

$$
\frac{2 \dot{S}}{\alpha}=\frac{1}{\alpha^{2}} u^{T} u-y^{T} y
$$

We are interested in the $L_{2}$ gain between the output and the disturbance, so we substitute $u=\left[\begin{array}{l}J d 1 \\ W d_{2}\end{array}\right]$ back into [15:

$$
\frac{2 \dot{S}}{\alpha}=\frac{1}{\alpha^{2}} d^{T}\left[\begin{array}{ll}
J & \\
& W
\end{array}\right]^{T}\left[\begin{array}{ll}
J & \\
& W
\end{array}\right] d-y^{T} y \leq \frac{\gamma^{2}}{\alpha^{2}} d^{T} d-y^{T} y
$$

where $\gamma^{2}$ is the greatest eigenvalue of the positive definite ma$\operatorname{trix} \operatorname{diag}(J, W)^{T} \operatorname{diag}(J, W)$. Using the formula for $J$ determined in section 2.2 it can be easily checked that

$$
\gamma^{2}=\max \left\{1, v_{1}^{2}, \ldots, v_{N}^{2}, \lambda_{1, \max }^{2}, \ldots, \lambda_{N, \max }^{2}\right\}
$$

where $d=\left[\begin{array}{ll}d_{1}^{T} & d_{2}^{T}\end{array}\right]^{T}$ and $\lambda_{i, \max }$ is the maximal eigenvalue of $W_{i}$.

Suppose that the modelling uncertainties can be represented by a nonlinear system $\Delta$ interconnected with (13) according to Fig. 1. By small gain theorem, if $\Delta$ has finite $L_{2}$ gain $\gamma_{\Delta}$ so that $\gamma_{\Delta} \frac{\gamma}{\alpha}<1$ and $\Delta$ is zero state detectable then the hierarchically controlled system remains globally stable even in the presence of uncertainty. (The zero state detectability of the controlled system (13), that is also necessary to apply the small gain theorem, follows from the fact that the invariant subset $\Omega=\left\{\left(\zeta_{1}, \zeta_{2}, \varepsilon_{3}\right) \mid \dot{S}\left(\zeta_{1}, \zeta_{2}, \varepsilon_{3}\right)=0\right\}$, examined in the previous section contains only the origin.)

\section{Formation control of road vehicles}

As an illustrative example we have solved in [7] a formation reconfiguration problem with five road vehicles. In the beginning the vehicles are in a column formation that is perpendicular to the trajectory. Then they are ordered to change their formation. The new formation is a line, which is tangential to the trajectory (according to Fig. 2). Of course, during the reconfiguration the vehicles must not collide and the entire group has to track a prescribed trajectory. The details of the controller design can be found in [7]. In this paper we focus on the robustness analysis only.

The vehicles were modelled by the simplified single-track dy- 

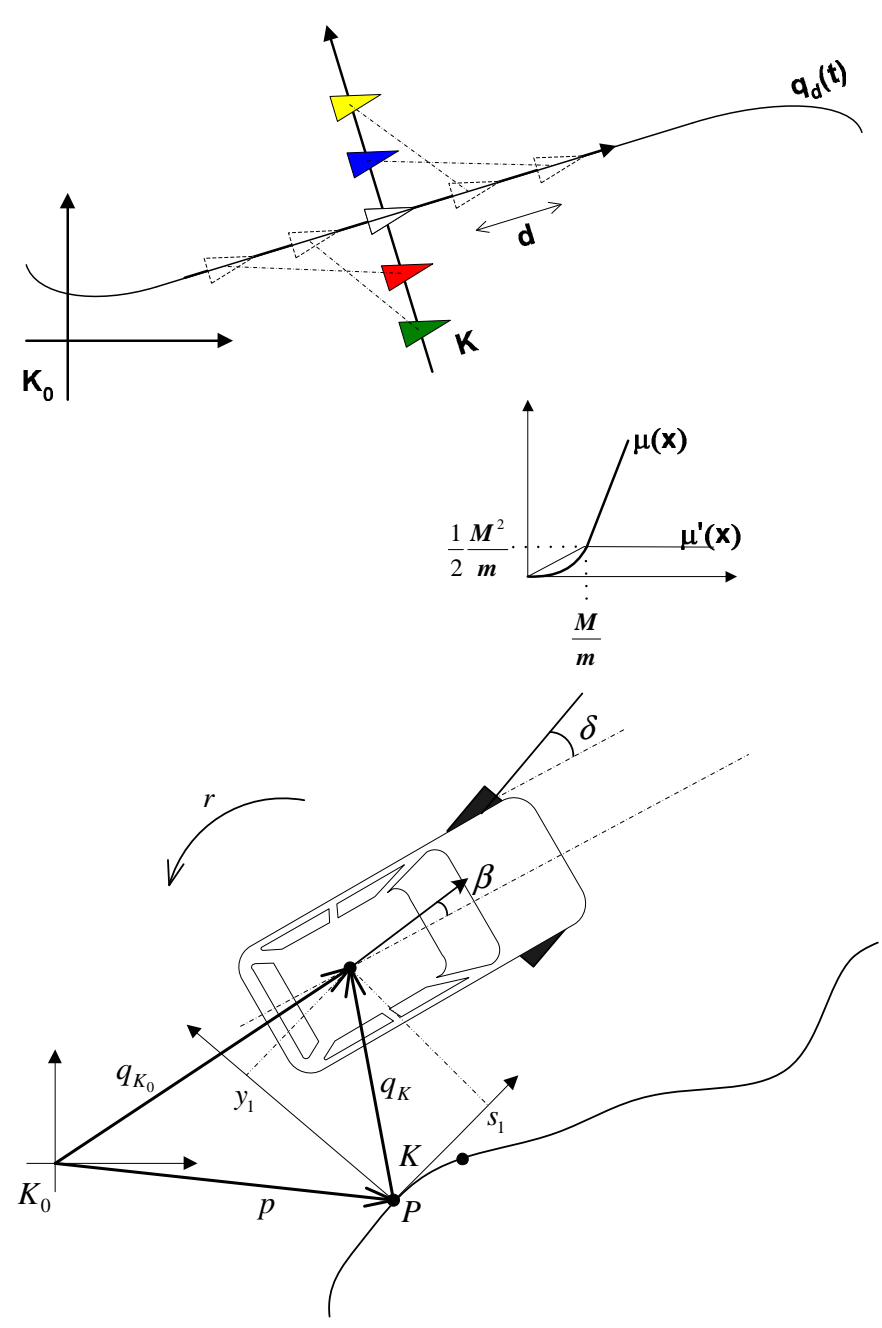

Fig. 2. Vehicle model and its parameters (above). Intended formation and scaling function $\mu(\cdot)$.

namics $([\overline{3}],[\overline{9}])$ given by the following equations:

$$
\begin{aligned}
& \dot{x}=v \cos (\beta+\psi)=v \cos (\phi) \\
& \dot{y}=v \sin (\beta+\psi)=v \sin (\phi) \\
& \dot{\phi}=\dot{\beta}+\dot{\psi}=\frac{a_{11}}{v} \beta+\frac{a_{12}}{v^{2}} r+\frac{b_{1}}{v} \delta \\
& \dot{\beta}=\frac{a_{11}}{v} \beta+\left(\frac{a_{12}}{v^{2}}-1\right) r+\frac{b_{1}}{v} \delta \\
& \dot{r}=a_{21} \beta+\frac{a_{22}}{v} r+b_{2} \delta \\
& \dot{v}=\alpha
\end{aligned}
$$

where $(x, y)$ denotes the position of the vehicle on the 2D plane in a fixed coordinate frame $K_{0}$ and $v, \beta, r, \psi$ are the velocity, slideslip angle, yaw-rate and orientation respectively (see Fig. 27. The control inputs are the steering angle $(\delta)$ and acceleration $(\alpha)$. As outputs the position coordinates $x$ and $y$ were chosen, both are supposed to be measured by appropriate inertial and/or GPS sensors. The remaining parameters of the model are constant and can be calculated as follows: $a_{11}=-\frac{c_{f}+c_{r}}{m}$, $a_{12}=\frac{c_{r} l_{r}-c_{f} l_{f}}{m}, a_{21}=\frac{c_{r} l_{r}-c_{f} l_{f}}{J}, a_{22}=-\frac{c_{r} l_{r}^{2}+c_{f} l_{f}^{2}}{J}, b_{1}=\frac{c_{f}}{m}$, $b_{2}=\frac{c_{f} l_{f}}{J}$, where $m$ is the mass of the vehicle, $c_{r}, c_{f}$ are the rear and front cornering stiffness, $J$ is the inertia, $l_{r}, l_{f}$ are the distances of the center of mass from the rear and front axle. This single-track dynamics describes well the vehicle motion in case of normal operation i.e. when the lateral acceleration is not too high $\left(<4 \frac{m}{s^{2}}\right)$. In [7] we have shown that this model can be transformed into a moving coordinate frame $K$ attached to a moving point $P$ of the reference trajectory. In the new coordinates 18 reads as

$$
\begin{array}{r}
\dot{s}_{1}=v \cos \theta-\dot{s}\left(1-c(s) y_{1}\right) \\
\dot{y}_{1}=v \sin \theta-c(s) \dot{s} s_{1} \\
\dot{\theta}=\dot{\phi}-\dot{\varphi}=\frac{a_{11}}{v} \beta+\frac{a_{12}}{v^{2}} r-c(s) \dot{s}+\frac{b_{1}}{v} \delta \\
\dot{v}=\alpha \\
\dot{\beta}=\frac{a_{11}}{v} \beta+\left(\frac{a_{12}}{v^{2}}-1\right) r+\frac{b_{1}}{v} \delta \\
\dot{r}=a_{21} \beta+\frac{a_{22}}{v} r+b_{2} \delta
\end{array}
$$

where $\theta=\phi-\varphi, s(t): \mathbb{R} \rightarrow \mathbb{R}$ is a continuous function, $\dot{p}=\left[\begin{array}{l}\dot{x}_{P}(t) \\ \dot{y}_{P}(t)\end{array}\right]=\left[\begin{array}{c}\dot{s} \cos \varphi(s) \\ \dot{s} \sin \varphi(s)\end{array}\right]$ defines the motion of $P$ on the trajectory curve and $c(s)=\frac{\partial \varphi(s)}{\partial s}$. By introducing new input and state variables so that

$$
x_{1}=\left[\begin{array}{l}
s_{1} \\
y_{1}
\end{array}\right] \quad x_{2}=\left[\begin{array}{l}
\theta \\
v
\end{array}\right] \quad x_{3}=\left[\begin{array}{l}
\beta \\
r
\end{array}\right] \quad u=\left[\begin{array}{l}
\delta \\
\alpha
\end{array}\right]
$$

the dynamics above can be rewritten in the form of (1). In the simulation the vehicles had the following identical modelling parameters, which were obtained by identifying a heavy-duty vehicle [9]:

$$
\begin{array}{lll}
a_{11}=-147.1481 & a_{12}=0.0645 & a_{21}=0.0123 \\
a_{22}=-147.1494 & b_{1}=66.2026 & b_{2}=31.9835
\end{array}
$$

If $1 \leq v \leq 25$ we found - by solving the appropriate LMI $[6]$ that the estimation error dynamics $\dot{e}_{3}=A_{3} e_{3}$ in (3) is quadratically stable with the following Lyapunov function

$$
W=e_{3}^{T}\left[\begin{array}{cc}
246.7608 & -4.7350 \\
-4.7350 & 247.7231
\end{array}\right] e_{3} \quad \forall i
$$

which makes it possible to use the dynamic inversion based controller.

Suppose now that the vehicle dynamics is uncertain and the uncertainty can be modelled by an appropriate dynamical system connected to the nominal vehicle model according to Fig. 11 In order to get some quantitative measurement on the robustness we have to determine the maximal $L_{2}$ gain $\gamma_{\Delta}$ of the potential disturbance models $\Delta$ that satisfies the small gain condition formulated in section 3 . It can be easily checked, that in our case

$$
\begin{aligned}
\alpha & =\min \left(k, \lambda^{*}\right)=k=1 \\
\gamma^{2} & =\max \left\{1, v_{1}^{2}, \ldots, v_{N}^{2}, \lambda_{1, \max }^{2}, \ldots, \lambda_{N, \max }^{2}\right\} \\
& =\max \left\{\lambda_{1, \max }^{2}, \ldots, \lambda_{N, \text { max }}^{2}\right\}=252^{2}
\end{aligned}
$$

This means that, if the $L_{2}$ gain of the disturbance model satisfies the relation $\gamma_{\Delta} \leq 1 / 252 \approx 0.004$ the cooperative system remains globally stable by means of the results of section 3 . 


\section{Conclusions}

In this paper the robustness analysis of the hierarchical formation control structure [7] has been performed. By exploiting the strict dissipativity of the passive components in the control structure we can determine the maximal $L_{2}$ gain of the uncertainty model at which the closed-loop system remains stable. The calculations have been demonstrated via a formation control problem of road vehicles.

\section{References}

1 Borrelli F, Keviczky T, Balas GJ, Collision-free UAV formation flight using decentralized optimization and invariant sets, Conference on Decision and Control, 2004, pp. 1099 - 1104.

2 Fax JA, Optimal and Cooperative Control of Vehicle Formations, California Institute of Technology, 2002. PhD thesis.

3 Kiencke U, Nielsen L, Automotive control system - for engine, driveline and vehicle, Springer, Berlin, 2000.

4 Olfati-Saber R, Flocking for Multi-Agent Dynamic Systems: Algorithms and Theory, IEEE Transactions on Automatic Control 51 (2006), no. 3, 401-420.

5 Olfati-Saber R, Murray RM, Distributed Cooperative Control of Multiple Vehicle Formations using Structural Potential Functions, The 15th IFAC World Congress , Barcelona, Spain, 2002.

6 Péni T, Bokor J, Dynamic inversion based velocity tracking control of road vehicles, NOLCOS, 6th IFAC Symposium on Nonlinear Control Systems (2004).

7 Péni T, Formation control of road vehicles based on dynamic inversion and passivity., Periodica Polytechnica Transportation Sciences (2008). Submitted.

8 Péni T, Bokor J, Formation stabilization of nonlinear vehicles based on dynamic inversion and passivity, 16th IFAC World Congress (2005).

9 Rödönyi G, Vehicle models for steering control, System and Control Laboratory, Computer and Automation Research Institute, 2003. SCL-4/2003.

10 Tanner HG, Jadbabaie A, Pappas GJ, Flocking in Fixed and Switching Networks, IEEE Transactions on Automatic Control 52 (2007), no. 5, 863868.

11 van der Schaft A, L2-Gain and Passivity Techniques in Nonlinear Control, Springer, Berlin, 2000.

12 Varaiya P, Smart Cars on Smart Roads: Problems of Control, IEEE Trans. on Automatic Control 38 (1993), no. 2, 195-207. 